\title{
Audio-visual navigation reduces treatment time in deep-inspiration breath hold radiotherapy
}

\author{
Martin Dolezel ${ }^{\mathrm{a}, \mathrm{b}, \mathrm{c}}$, Karel Odrazka ${ }^{\mathrm{b}, \mathrm{c}, \mathrm{d}}$, Jaroslav Vanasek ${ }^{\mathrm{b}}$, Igor Hartmann ${ }^{\mathrm{e}}$, Tereza Kohlova ${ }^{\mathrm{f}}$, Zuzana Vlachova ${ }^{\mathrm{a}}$
}

\begin{abstract}
Aims. The purpose of this study was to compare the treatment times for deep-inspiration breath hold with and without audio-visual (A-V) navigation.

Methods. We measured the real treatment time in 60 patients with breast cancer undergoing postoperative radiotherapy. Thirty consecutive patients were treated without deep-inspiration breath hold (DIBH) and another 30 patients using deep-inspiration breath hold (10 patients with DIBH only, 10 patients with DIBH using visual feedback and 10 patients with DIBH using visual feedback following breath training). The treatment time was relativized to number of fields and 100 monitor units (MU). The independent t-test was used to analyse differences between cohorts.

Results. The mean treatment time for patients without DIBH was 46.5 seconds per field and 90.9 seconds per $100 \mathrm{MU}$, for DIBH only 92.3 and 170.2 seconds, for DIBH with audio-visual navigation 68.1 and 133.8 seconds, and for DIBH with A-V feedback including breath training 66.1 and 132.5 seconds. The treatment times for patients treated using DIBH with visual navigation were significantly shorter in comparison with patients without visual feedback. We were not able to prove any significant benefit for breath training in terms of reducing the treatment time.
\end{abstract}

Conclusion. Audio-visual navigation enables to significantly reduce the treatment time in comparison with DIBH without A-V feedback.

Key words: deep-inspiration breath hold, breast cancer, audio-visual navigation, treatment time

Received: July 26, 2019; Revised: September 24, 2019; Accepted: September 26, 2019; Available online: October 10, 2019 https://doi.org/10.5507/bp.2019.051

() 2020 The Authors; https://creativecommons.org/licenses/by/4.0/

${ }^{a}$ Department of Oncology, Faculty of Medicine and Dentistry, Palacky University Olomouc and University Hospital Olomouc, Czech Republic ${ }^{b}$ Oncology Centre, Multiscan and Pardubice Regional Hospital, Pardubice, Czech Republic

'First Faculty of Medicine, Charles University, Prague, Czech Republic

${ }^{d}$ Third Faculty of Medicine, Charles University, Prague, Czech Republic

${ }^{e}$ Department of Urology, Faculty of Medicine and Dentistry, Palacky University Olomouc and University Hospital Olomouc, Czech Republic ${ }^{f}$ Department of Radiotherapy and Oncology, Charles University and Královské Vinohrady University Hospital, Prague, Czech Republic Corresponding author: Martin Dolezel, e-mail:dolezelm@email.cz

\section{INTRODUCTION}

Breast cancer is the most common cancer and the leading cause of cancer death among women worldwide. Whole breast radiotherapy as part of breast-conserving therapy has been shown to be an appropriate option to radical mastectomy for many women with early-stage breast cancer ${ }^{1-3}$.

However, the old meta-analyses and registry-based studies reported higher mortality rates for patients undergoing radiotherapy of left-sided cancers compared with right-sided tumors ${ }^{1,47}$. A prospective cohort study of about 300,000 women in US Surveillance, Epidemiology, and End Results (SEER) cancer registries to evaluate the longterm mortality from heart disease after RT for early breast cancer indicated a reduction in any early cardiac mortality ratios (left versus right tumor) in women diagnosed during 1983-92 in comparison to 1973-82 (ref. ${ }^{8}$ ). These results may reflect improvements in treatment planning of breast radiotherapy.

The dose-effect relationship based on the mean heart dose (MHD) to the whole heart was demonstrated by Darby et $\mathrm{al}^{9}$. The authors found a relative increase of $7.4 \%$ per Gy of MHD in the rate of major acute coronary events (ACEs). The findings were recently validated by Bogaard et al. using an independent cohort of consecutive patients based on individual three-dimensional (3D) dose distributions derived from computed tomography (CT) planning scans. The authors demonstrated an increase of $16.5 \%$ in the cumulative incidence of AEs per Gy of radiation to the whole heart in the first 9 years after treatment ${ }^{10}$. There are several techniques to decrease cardiac dose including intensity modulated radiotherapy, prone positioning, and respiratory gating.

At our institution, a deep-inspiration breath hold (DIBH) technique is used for all patients with left-sided breast cancer treated with whole breast radiotherapy. We implemented for DIBH visual navigation to maximize patient comfort and reduce the treatment time. The purpose of this study was to compare the treatment times for DIBH with and without visual navigation. 


\section{PATIENTS AND METHODS}

We measured prospectively the real treatment time in 60 consecutive patients with breast cancer treated with postoperative radiotherapy. Thirty consecutive patients were treated without DIBH and another 30 patients using deep-inspiration breath hold (10 patients with DIBH only, 10 patients with DIBH using visual feedback and 10 patients with DIBH using visual feedback including breath training). All patients signed informed consent.

All were scanned on a supine breast board with left arm extended above the head resting on supports. The clinical target volume (CTV) included all remaining breast tissue (including regional nodes when indicated), the margin for the planning target volume was $10 \mathrm{~mm}$ in all cases. The window amplitude was $5 \mathrm{~mm}$ for patients treated using DIBH. The patients were treated with 3D CRT using 4 tangential fields ( $6 \mathrm{MeV}$ and $18 \mathrm{MeV}$ ) and mostly 1-2 additional fields to achieve homogenous plans. The prescribed dose was $48.6 \mathrm{~Gy}$ in 27 fractions ( $1.8 \mathrm{~Gy}$ per day).
The patients with DIBH alone were instructed during the treatment only by voice. For patients with visual navigation our in-house system was used. This works by splitting and amplifying the video output signal directly from the Varian Real-Time Position Management (RPM) workstation or TrueBeam Respiratory Motion Management (RMM) workstation (Varian Medical Systems, Palo Alto, CA) into two signals. The first signal is connected to the monitor in the operator room. The second signal is linked to the video scaler to choose the visual part of navigation. The signal is converted from DVI to HDMI. This information is transformed to local area network using HDMI extender. The last step was splitting the signal to different displays in the treatment room according to patient's preference.

The last group (patients with DIBH using visual feedback including breath training) underwent a meeting with radiation therapist one week before planning CT. Patients were instructed to practice deep inspiration breath hold at home - lying on the back, one hand behind head, a deep breath in the chest (not in the abdomen) watching the chest rising, a comfortable breath holding.

Table 1. Treatment times for each fraction related to field.

\begin{tabular}{|c|c|c|c|c|c|c|c|c|}
\hline \multirow[b]{2}{*}{ Fr. } & \multicolumn{2}{|c|}{ W/out DIBH } & \multicolumn{2}{|c|}{ DIBH (w/out A-V) } & \multicolumn{2}{|c|}{ DIBH (A-V with T) } & \multicolumn{2}{|c|}{ DIBH (A-V w/out T) } \\
\hline & Mean (s) & Range (s) & Mean (s) & Range (s) & Mean (s) & Range (s) & Mean (s) & Range (s) \\
\hline 1 & 48.1 & $(43.2-58.8)$ & 107.5 & $(75.6-174)$ & 89.3 & $(58.8-157.8)$ & 90.4 & $(64.2-144)$ \\
\hline 2 & 44.1 & $(33-55.8)$ & 144.0 & $(66-272.4)$ & 69.3 & $(56.4-91.8)$ & 69.7 & $(58.8-97.8)$ \\
\hline 3 & 50.0 & $(41.4-67.2)$ & 99.6 & $(66.6-162.6)$ & 67.8 & $(51.6-95.4)$ & 68.8 & $(54-93)$ \\
\hline 4 & 47.2 & $(42.6-57)$ & 131.3 & $(61.8-194.4)$ & 65.3 & $(48-89.4)$ & 66.2 & $(51.6-84.6)$ \\
\hline 5 & 44.9 & $(38.4-55.2)$ & 84.2 & $(68.4-106.8)$ & 68.3 & $(54.6-81)$ & 68.3 & $(54.6-96.6)$ \\
\hline 6 & 46.7 & $(36.6-57.6)$ & 88.9 & $(60.6-141.6)$ & 68.0 & $(47.4-87)$ & 70.2 & $(59.4-83.1)$ \\
\hline 7 & 46.2 & $(37.2-58.2)$ & 90.6 & $(67.2-129.6)$ & 64.4 & $(48.6-84.6)$ & 67.7 & $(58.2-93.6)$ \\
\hline 8 & 44.4 & $(33.6-51)$ & 96.5 & $(58.8-123.6)$ & 64.3 & $(50.4-104.4)$ & 67.3 & $(54.6-85.2)$ \\
\hline 9 & 50.4 & $(36.6-96)$ & 88.0 & $(58.2-114.6)$ & 62.7 & $(49.8-78)$ & 63.3 & $(55.8-73.2)$ \\
\hline 10 & 47.1 & $(42-58.8)$ & 87.5 & $(59.4-111)$ & 68.1 & $(53.4-95.4)$ & 69.2 & $(57.6-80.4)$ \\
\hline 11 & 48.1 & $(43.2-55.2)$ & 90.3 & $(75-126.6)$ & 59.5 & $(46.8-76.2)$ & 66.1 & $(55.8-76.2)$ \\
\hline 12 & 48.1 & $(36.6-69)$ & 101.5 & $(77.4-165.6)$ & 62.9 & $(36-98.4)$ & 74.6 & $(55.2-93.6)$ \\
\hline 13 & 45.5 & $(36.7-58.2)$ & 83.4 & $(66-105.6)$ & 58.3 & $(36.6-73.8)$ & 57.8 & $(41.4-66)$ \\
\hline 14 & 44.5 & $(36-50.4)$ & 93.2 & $(59.4-133.8)$ & 65.4 & $(53.4-93)$ & 68.5 & $(46.8-92.4)$ \\
\hline 15 & 45.4 & $(37.2-58.8)$ & 84.3 & $(61.2-117.6)$ & 76.4 & $(48.6-156.6)$ & 67.9 & $(54-103.2)$ \\
\hline 16 & 48.5 & $(36-76.2)$ & 80.4 & $(60-121.8)$ & 66.5 & $(49.2-96)$ & 68.6 & $(52.2-103.8)$ \\
\hline 17 & 43.9 & $(33.6-50.4)$ & 91.9 & $(34.8-120.6)$ & 69.4 & $(52.2-94.8)$ & 67.2 & $(50.4-75)$ \\
\hline 18 & 46.6 & $(38.4-57)$ & 89.2 & $(59.4-168.6)$ & 69.3 & $(39.6-120)$ & 61.5 & $(56.4-66)$ \\
\hline 19 & 45.9 & $(38.4-56.4)$ & 91.5 & $(67.2-122.4)$ & 61.6 & $(46.8-95.4)$ & 64.5 & $(52.8-82.8)$ \\
\hline 20 & 45.0 & $(36.6-56.3)$ & 86.8 & $(54.6-143.4)$ & 65.9 & $(49.2$ - 99) & 63.9 & $(54-87)$ \\
\hline 21 & 51.5 & $(36-108)$ & 86.3 & $(68.4-125.4)$ & 63.4 & $(40,2-118,8)$ & 64.1 & $(48.6-94.2)$ \\
\hline 22 & 44.3 & $(35.4-56.7)$ & 78.2 & $(57-98.4)$ & 63.1 & $(40-90)$ & 74.2 & $(37.2-160.2)$ \\
\hline 23 & 47.5 & $(36.6-58.8)$ & 86.0 & $(59.4-115.8)$ & 69.5 & $(48-98.4)$ & 67.2 & $(53.4-86.4)$ \\
\hline 24 & 44.8 & $(37.2-51)$ & 82.1 & $(65.4-97.2)$ & 62.0 & $(47.4-73.8)$ & 66.7 & $(57-88.8)$ \\
\hline 25 & 44.7 & $(36-56.4)$ & 79.1 & $(57.6-105)$ & 61.7 & $(37.8-88.8)$ & 68.6 & $(52.8-98.4)$ \\
\hline 26 & 45.3 & $(37.2-51.6)$ & 86.3 & $(77.4-97.8)$ & 64.0 & $(45-96.6)$ & 67.6 & $(48.6-123)$ \\
\hline 27 & 46.0 & $(33.6-55.2)$ & 84.7 & $(57.6-121.8)$ & 59.3 & $(37.2-75)$ & 68.1 & $(57.6-90.6)$ \\
\hline $1-27$ & 46.5 & & 92.3 & & 66.1 & & 68.1 & \\
\hline
\end{tabular}

w/out DIBH - patients without deep-inspiration breath hold technique; DIBH (w/out A-V) - patients with deep-inspiration breath hold technique only; DIBH (A-V with T) - patients with DIBH and visual feedback including breath training; DIBH (A-V w/out T) - patients with deep-inspiration breath hold technique including visual navigation; s - second 
Table 2. Treatment times for each fraction related to $100 \mathrm{MU}$.

\begin{tabular}{|c|c|c|c|c|c|c|c|c|}
\hline & \multicolumn{2}{|c|}{ W/out DIBH } & \multicolumn{2}{|c|}{ DIBH (w/out AV) } & \multicolumn{2}{|c|}{ DIBH (AV with T) } & \multicolumn{2}{|c|}{ DIBH (AV w/out T) } \\
\hline & $\begin{array}{l}\text { Mean } \\
\text { (s) }\end{array}$ & $\begin{array}{l}\text { Range } \\
\text { (s) }\end{array}$ & $\begin{array}{l}\text { Mean } \\
\text { (s) }\end{array}$ & $\begin{array}{l}\text { Range } \\
\text { (s) }\end{array}$ & $\begin{array}{l}\text { Mean } \\
\text { (s) }\end{array}$ & $\begin{array}{l}\text { Range } \\
\text { (s) }\end{array}$ & $\begin{array}{l}\text { Mean } \\
\text { (s) }\end{array}$ & $\begin{array}{l}\text { Range } \\
\text { (s) }\end{array}$ \\
\hline 1 & 94.1 & $(74.6-118.8)$ & 205.9 & $(116.6-358.7)$ & 179.4 & $(100.7-308.4)$ & 159.6 & $(112.9-246)$ \\
\hline 2 & 86.5 & $(57.6-111.6)$ & 250.3 & $(186.2-337.5)$ & 140.2 & $(93.4-174.4)$ & 135.1 & $(98.6-167.8)$ \\
\hline 3 & 97.7 & $(76.8-129.6)$ & 182.7 & $(117.5-253.3)$ & 134.7 & $(110.9-155.6)$ & 134.2 & $(90.7-187.7)$ \\
\hline 4 & 93.6 & $(73.8-152.4)$ & 225.3 & $(69.5-400.1)$ & 129 & $(111.6-140.1)$ & 129.7 & $(86.6-192.4)$ \\
\hline 5 & 87.9 & $(66-111)$ & 161.1 & $(95.1-220.7)$ & 137.4 & $(112.1-176.5)$ & 131.9 & $(98.9-164.9)$ \\
\hline 6 & 92.2 & $(64.2-119.4)$ & 160 & $(149.1-170.9)$ & 137.2 & $(101.2-196.1)$ & 137 & $(98.9-169.1)$ \\
\hline 7 & 90.9 & $(64.8-125.4)$ & 166.6 & $(136.5-189.4)$ & 130.6 & $(89.8-236)$ & 131.3 & $(101.9-195.4)$ \\
\hline 8 & 87.3 & $(58.8-108)$ & 177.6 & $(129.8-216)$ & 132.4 & $(97.4-278.2)$ & 131.5 & $(94.6-193.7)$ \\
\hline 9 & 98.4 & $(64.2-184.2)$ & 162.6 & $(120.4-201.1)$ & 127.5 & $(92.1-196.1)$ & 124.3 & $(95.2-166.4)$ \\
\hline 10 & 91.7 & $(69-119.4)$ & 162.5 & $(116.9-222.1)$ & 138.4 & $(94.3-217.9)$ & 136 & $(96.7-183.6)$ \\
\hline 11 & 93.1 & $(70.8-114.6)$ & 169.1 & $(128.4-219.4)$ & 118.8 & $(101.1-132.5)$ & 129.1 & $(91.5-170.8)$ \\
\hline 12 & 93.4 & $(75.6-121.2)$ & 195 & $(104.8-341.6)$ & 126.5 & $(71.8-234.5)$ & 145.1 & $(107.4-196)$ \\
\hline 13 & 88.9 & $(70.2-117)$ & 155.2 & $(110.9-185.9)$ & 116.2 & $(82.7-144.8)$ & 113 & $(81.7-146.2)$ \\
\hline 14 & 87 & $(63-101.4)$ & 173.1 & $(119.1-275.6)$ & 131.2 & $(97.7-192.2)$ & 132.4 & $(92.6-164.5)$ \\
\hline 15 & 89 & $(64.6-118.5)$ & 154.6 & $(123.5-177.4)$ & 151.7 & $(89.2-265)$ & 134.1 & $(93.7-235.7)$ \\
\hline 16 & 94.5 & $(63.5-146.7)$ & 146.9 & $(128-169.8)$ & 133.8 & $(93.4-199.9)$ & 132.8 & $(87.1-194.6)$ \\
\hline 17 & 85.8 & $(58.8-97.8)$ & 171.3 & $(71.3-208.2)$ & 139.4 & $(91.8-189.1)$ & 135.4 & $(84.2-189)$ \\
\hline 18 & 91.2 & $(72.6-116.6)$ & 157.1 & $(124.1-191.6)$ & 137.3 & $(99.7-250)$ & 120.2 & $(92.3-151.1)$ \\
\hline 19 & 89.8 & $(66.6-113)$ & 171 & $(128.3-228)$ & 121.9 & $(94-146.7)$ & 127.2 & $(87.2-189.1)$ \\
\hline 20 & 88.3 & $(63.9-113,6)$ & 155.2 & $(116-184.1)$ & 131.2 & $(100.1-147.1)$ & 125.1 & $(90.5-162.7)$ \\
\hline 21 & 100.3 & $(63.4-190,7)$ & 160.3 & $(132.1-224.5)$ & 124.8 & $(73.2-183)$ & 125 & $(94.9-197.3)$ \\
\hline 22 & 86.7 & $(65.5-112.7)$ & 145.9 & $(103.3-178)$ & 125.5 & $(95.9-176.2)$ & 142.4 & $(84.4-299.7)$ \\
\hline 23 & 92.5 & $(71.3-118.5)$ & 161 & $(122-222.7)$ & 139.1 & $(92.7-191.9)$ & 133.2 & $(87.7-192.3)$ \\
\hline 24 & 87.9 & $(64.9-113.6)$ & 158 & $(90.4-208.6)$ & 124.9 & $(94.6-153.8)$ & 129.6 & $(98.4-165.7)$ \\
\hline 25 & 87.4 & $(66.2-113)$ & 146.1 & $(110.6-199.6)$ & 124.4 & $(86.3-185)$ & 134.9 & $(87.8-224.3)$ \\
\hline 26 & 88.4 & $(67.9-102,5)$ & 167.2 & $(103-251.6)$ & 127.3 & $(90.9-165.6)$ & 133.1 & $(83.1-241.4)$ \\
\hline 27 & 90.1 & $(58.44-110)$ & 153.6 & $(123.6-185.4)$ & 117.5 & $(99.2-151.9)$ & 131.6 & $(109.9-261.5)$ \\
\hline $1-27$ & 90.9 & & 170.2 & & 132.5 & & 133.8 & \\
\hline
\end{tabular}

w/out DIBH - patients without deep-inspiration breath hold technique; DIBH (w/out A-V) - patients with deep-inspiration breath hold technique only; DIBH (A-V with T) - patients with DIBH and visual feedback including breath training; DIBH (A-V w/out T) - patients with deep-inspiration breath hold technique including visual navigation; s - second; MU - monitor unit

All patients underwent on-line correction of treatment position using two orthogonal $\mathrm{kV}$ images prior to every fraction of radiotherapy. We monitored the actual time from the first $\mathrm{kV}$ image to the end of the treatment fraction. To reduce variability introduced by the human process of image matching we excluded time between second $\mathrm{kV}$ and the shift of the table needed for evaluation and correction of set-up errors. The treatment time was relativized to number of fields and $100 \mathrm{MU}$. We compared the treatment time for these four cohorts: patients without deep-inspiration breath hold technique, patients with DIBH only, patients with DIBH including visual navigation and patients with DIBH and visual feedback including breath training.

The independent t-test was used to analyse differences between cohorts. All $P$-values are two-sided and are not adjusted for multiple comparisons. Comparisons with $P$-values of $<0.05$ were considered significant.

The last evaluation consisted of determining the rate of automatic irradiation interruption due to incorrect chest position of the patient. We monitored prospectively each treatment fraction throughout the course of radio- therapy in all patients. Every automatic interruption of irradiation due to inadequate chest position was recorded. Subsequently, the ratio of the protracted fractions to the total number of fractions was determined for each cohort of patients treated using deep-inspiration breath hold radiotherapy.

\section{RESULTS}

A total of 60 patients were included in this study, which represented 1620 treatment fractions. Tables 1 and 2 contain the treatment times for each fraction related to field (Table 1) and per each 100 MU (Table 2).

The mean treatment time for patients without DIBH was $46.5 \mathrm{~s}$ per field and $90.9 \mathrm{~s}$ per $100 \mathrm{MU}$, for DIBH only 92.3 and $170.2 \mathrm{~s}$, for DIBH with audio-visual navigation 68.1 and $133.8 \mathrm{~s}$, and for DIBH with A-V feedback including breath training 66.1 and $132.5 \mathrm{~s}$.

The treatment times for patients treated using DIBH with visual navigation were significantly shorter than for those without visual feedback. We were not able to con- 


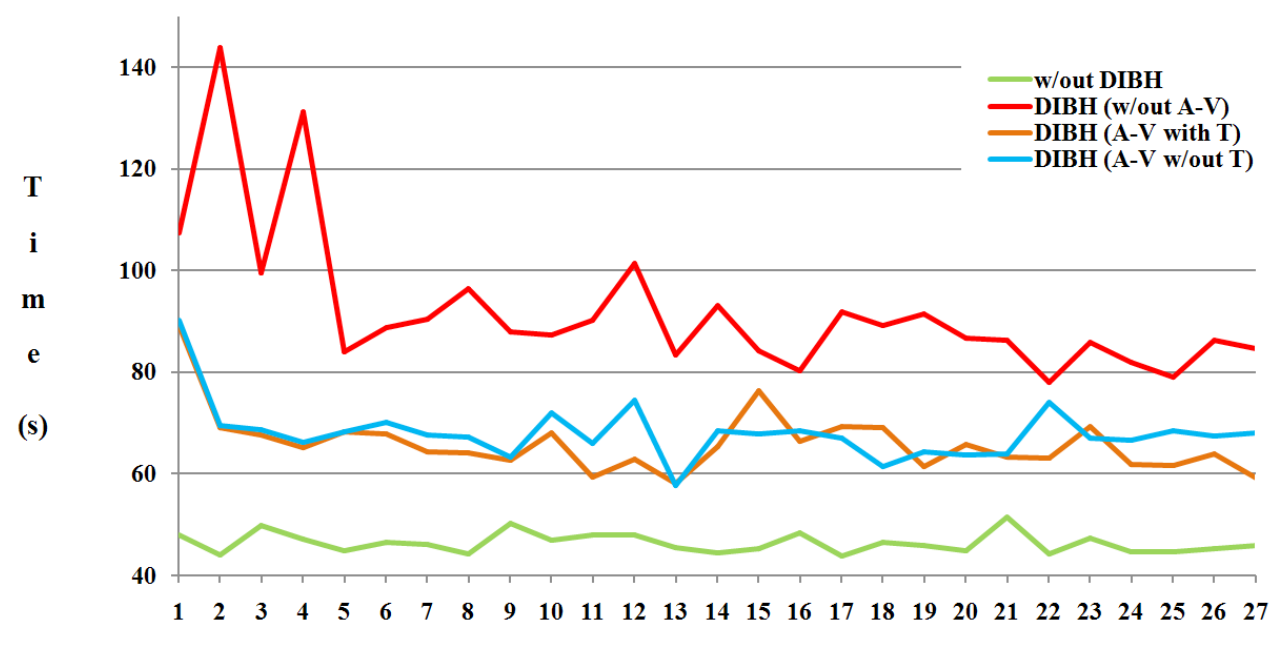

F r a c t i o n

\begin{abstract}
Abbreviations:
w/out DIBH $\cong$ patients without deep-inspiration breath hold technique;

DIBH (w/out A-V) $\cong$ patients with deep-inspiration breath hold technique only;

DIBH (A-V with T) $\cong$ patients with DIBH and visual feedback including breath training;

DIBH $(\mathbf{A}-\mathbf{V}$ w/out T) $\cong$ patients with deep-inspiration breath hold technique including visual navigation;

$\mathbf{S} \cong$ second
\end{abstract}

Fig. 1. Treatment times for each fraction converted per field.



Abbreviations:

w/out DIBH $\cong$ patients without deep-inspiration breath hold technique

DIBH (w/out $\mathbf{A}-\mathbf{V}) \cong$ patients with deep-inspiration breath hold technique only;

DIBH (A-V with T) $\cong$ patients with DIBH and visual feedback including breath training;

DIBH $(A-V$ w/out $\mathbf{T}) \cong$ patients with deep-inspiration breath hold technique including visual navigation;

$\mathbf{M U} \cong$ monitor unit; $\mathbf{s} \cong$ second

Fig. 2. Treatment times for each fraction converted per $100 \mathrm{MU}$.

firm any significant benefit of breath training in terms of reducing the treatment time.

We also investigated the duration of treatment fraction at different phases (weeks) of radiotherapy (Fig. 1 and 2). There were no significant differences in any week of treatment during radiotherapy without DIBH or irradiation with DIBH using audio-visual navigation. The treatment times of individual fractions were consistent throughout whole treatment cycle. Nevertheless, we observed the shortening of irradiation times with an increasing number of fractions in patients undergoing DIBH radiotherapy without audio-visual feedback. The difference between the first two weeks and the last two weeks of the treatment course were statistically significant $(P=0.02)$. 
Finally, we determined the rate of automatic irradiation interruption due to incorrect chest position of the patient. The treatment session was prolonged in $9.6 \%$ of fractions in patients with DIBH using visual feedback, in $10.7 \%$ of fractions with DIBH using visual feedback including breath training, and in $60.7 \%$ of treatment fractions using deep-inspiration breath hold only. Moreover, we detected that in patients with audio-visual feedback, the treatment sessions were interrupted repeatedly in only $0.7 \%$ and $1.1 \%$ of treatment fractions. Unfortunately, a repeated discontinuation of the irradiation was found in $14.8 \%$ of treatment sessions without audio-visual navigation.

\section{DISCUSSION}

Cardiovascular disease and breast cancer are significant causes of morbidity and mortality in women. Therefore a multidisciplinary approach is essential. The American Heart Association warns that cancer outcomes can be influenced by cardiovascular health, and cancer treatment itself can result in cardiovascular toxicity ${ }^{11}$.

There is growing evidence from retrospective and planning studies demonstrating a significant reduction in dose to the heart (MHD and left anterior descending coronary artery) in patients with left-sided breast cancers undergoing deep-inspiration breath hold radiotherapy ${ }^{12-14}$. DIBH irradiation significantly reduces MHD using both IMRT and VMAT techniques compared with free-breathing radiation $^{15}$. Corradini et al. published the results of a comparison between 3D-CRT and VMAT with free-breathing or DIBH. The authors conclude that 3D-CRT plans using DIBH leads to the lowest risk of both major coronary events and secondary lung cancer ${ }^{16}$. There are significant problems associated with heart sparing still to be solved, including contouring OAR and selection of ideal treatment technique (what heart sub-volumes are the most important and how to contour them, what are the appropriate dose constraints, how to select optimal treatment technique) (ref. ${ }^{15,17,18}$ ).

There are currently a number of studies underway concerning the reduction of cardiac toxicity including UK HeartSpare Study ${ }^{19}$.

Despite all these ambiguities, DIBH is explicitly recommended for minimizing the heart dose in the last Executive summary of an American Society for Radiation Oncology evidence-based guideline for radiation therapy for the whole breast ${ }^{20}$.

Unfortunately, this technique can lead to disproportionate prolongation of treatment time for each fraction. To reduce the actual treatment time and to improve patient comfort, we devised an audio-visual system and introduced it into daily practice. This study focused on the evaluation of treatment times for different DIBH approaches.

To the best of our knowledge, this is the first study investigating the time factor associated with different forms of DIBH. We have shown that audio-visual navigation re- duces the treatment time. However, the absolute saving of time using this technique is limited - on the edge of clinical benefit. We are convinced that the improvement of patients' comfort during treatment may be more important. Quantification of this benefit will be the subject of further research.

It is interesting that we found no difference in treatment time between a group with $\mathrm{A}-\mathrm{V}$ navigation with breath training and A-V feedback itself. A-V feedback allows patients to interactively change the actual chest position as required. We are convinced that an option of the interactive changes of chest position compensate suboptimal breathing excursion. Moreover, due to strict amplitude window $5 \mathrm{~mm}$ we can reduce PTV margins from $10 \mathrm{~mm}$ to $5-7 \mathrm{~mm}$. This can lead to further sparing of organs at risk.

Radiation therapy is rapidly evolving and modern techniques such as DIBH and stereotactic radiotherapy are often time consuming. Due to a rapidly changing health care environment, we are faced with increasing numbers of patients in our radiotherapy departments. We are convinced that rational logistics in treatment delivery plays an increasing role these days. Hence significant reduction in treatment time and better patient convenience should be one of our goals.

The simplicity of the procedure and an easy understanding of the principle of this innovation both for patients and staff has enabled us to rapidly implement the audio-visual navigation into a practical treatment protocol. At present, all patients with left-sided breast cancer are treated at our department using DIBH with A-V feedback.

\section{CONCLUSION}

Our data show that audio-visual navigation enables us to reduce the treatment time in comparison with deep inspiration breath hold without $\mathrm{A}-\mathrm{V}$ feedback.

Acknowledgement: This work was supported by Project AZV No. 17-29389A.

Author contributions: JS, IH, TK, ZV: literature search; $\mathrm{MD}, \mathrm{JV}, \mathrm{KO}$ : data analysis; $\mathrm{MD}, \mathrm{KO}$ : manuscript writing and final approval.

Conflict of interest statement: The authors state that there are no conflicts of interest regarding the publication of this article.

\section{REFERENCES}

1. Early Breast Cancer Trialists' Collaborative Group (2000) Favourable and unfavourable effects on long-term survival of radiotherapy for early breast cancer: an overview of the randomised trials. Lancet 355:1757-70.

2. Gasparini G, Panizzoni GA, Dal Fior S, Germani B, Dall'Antonia F, Segato G, Meli S, Pozza F. Conservative surgery and irradiation (QUART) in the treatment of 243 stage I-II breast cancer patients. Anticancer Res 1991;11:1635-40.

3. Rose MA, Olivotto I, Cady B, Koufman C, Osteen R, Silver B, Recht A, Harris JR. Conservative surgery and radiation therapy for early breast cancer long term cosmetic results. Arch Surg 1989;124:153-7. 
4. Melichar B, Doležal J, Srámek V, Kalábová H, Krčmová LK, Hyšpler $R$ Studentová $H$, Vošmik M, Pecka M, Svobodník A, Pecen L, Doležel $\mathrm{M}$, Solichová D. Prevalence of perfusion defects detected by stress 99mtechnetium sestamibi myocardial perfusion single-photon emission computed tomography in asymptomatic patients with breast cancer. Anticancer Res 2014;34(7):3689-94.

5. Rutqvist LE, Johansson $\mathrm{H}$. Mortality by laterality of the primary tumour among 55,000 breast cancer patients from the Swedish Cancer Registry. Br J Cancer 1990;61:866-8.

6. Paszat LF, Mackillop WJ, Groome PA, Schulze K, Holowaty E. Mortality from myocardial infarction after adjuvant radiotherapy for breast cancer in the Surveillance, Epidemiology, and End-Results cancer registries. J Clin Oncol 1998;16:2625-31.

7. McGale P, Darby SC, Hall P, Adolfsson J, Bengtsson NO, Bennet AM Fornander T, Gigante B, Jensen MB, Peto R, Rahimi K, Taylor CW, Ewertz M. Incidence of heart disease in 35,000 women treated with radiotherapy for breast cancer in Denmark and Sweden. Radiother Oncol 2011;100:167-75.

8. Darby SC, McGale P, Taylor CW, Peto R. Long-term mortality from heart disease and lung cancer after radiotherapy for early breast cancer: prospective cohort study of about 300,000 women in US SEER cancer registries. Lancet Oncol 2005;6:557-65.

9. Darby SC, Ewertz M, McGale P, Bennet AM, Blom-Goldman U, Brønnum D, Correa C, Cutter D, Gagliardi G, Gigante B, Jensen MB, Nisbet A, Peto R, Rahimi K, Taylor C, Hall P. Risk of ischemic heart disease in women after radiotherapy for breast cancer. N Engl J Med 2013;368:987-98.

10. Van Den Bogaard VA, Ta BD, Van Der Schaaf A, Bouma AB, Middag AM, Bantema-Joppe EJ, van Dijk LV, van Dijk-Peters FB, Marteijn LA, de Bock GH, Burgerhof JG, Gietema JA, Langendijk JA, Maduro $\mathrm{JH}$, Crijns AP. Validation and Modification of a Prediction Model for Acute Cardiac Events in Patients With BreastCancer Treated With Radiotherapy Based on Three-Dimensional Dose Distributions to Cardiac Substructures. J Clin Oncol 2017;35(11):1171-8.

11. Mehta LS, Watson KE, Barac A, Beckie TM, Bittner V, Cruz-Flores S, Dent S, Kondapalli L, Ky B, Okwuosa T, Piña IL, Volgman AS Cardiovascular Disease and Breast Cancer: Where These Entities Intersect: A Scientific Statement From the American Heart Association. Circulation 2018; 20:137(8) e30-e66.
12. Hepp R, Ammerpohl M, Morgenstern C, Nielinger L, Erichsen $P$, Abdallah A, Galalae R. Deep inspiration breath-hold (DIBH) radiotherapy in left-sided breast cancer: Dosimetrical comparison and clinical feasibility in 20 patients. Strahlenther Onkol 2015;191(9):7106.

13. Stranzl H, Zurl B. Postoperative irradiation of left-sided breast cancer patients and cardiac toxicity. Does deep inspiration breathhold (DIBH) technique protect the heart? Strahlenther Onkol 2008;184(7):354-8. doi: 10.1007/s00066-008-1852-0

14. Lawler G, Leech M. Dose Sparing Potential of Deep Inspiration Breathhold Technique for Left Breast Cancer Radiotherapy Organs-at-risk. Anticancer Res 2017;37:883-90. doi: 10.21873/anticanres.11394

15. Sakka M, Kunzelmann L, Metzger M, Grabenbauer GG. Cardiac dose-sparing effects of deep-inspiration breath-hold in left breast irradiation: Is IMRT more beneficial than VMAT? Strahlenther Onkol 2017;193(10):800-11.

16. Corradini $\mathrm{S}$, Ballhausen $\mathrm{H}$, Weingandt $\mathrm{H}$, Freislederer $\mathrm{P}$, Schönecker S, Niyazi M, Simonetto C, Eidemüller M, Ganswindt U, Belka C. Leftsided breast cancer and risks of secondary lung cancer and ischemic heart disease: Effects of modern radiotherapy techniques. Strahlenther Onkol 2018; 194(3):196-205

17. Duma MN, Herr AC, Borm KJ, rott KR, Molls M, Oechsner M, Combs SE. Tangential Field Radiotherapy for Breast Cancer-The Dose to the Heart and Heart Subvolumes: What Structures Must Be Contoured in Future Clinical Trials? Front Oncol 2017;19;7:130.

18. Munshi A, Khataniar N, Sarkar B, Bera ML, Mohanti BK. Spatial orientation of coronary arteries and its implication for breast and thoracic radiotherapy-proposing "coronary strip" as a new organ at risk. Strahlenther Onkol 2018;194(8):711-8.

19. Bartlett FR, Donovan EM, McNair HA, Corsini LA, Colgan RM, Evans PM, Maynard L, Griffin C, Haviland JS, Yarnold JR, Kirby AM. The UK HeartSpare Study (Stage II): Multicentre Evaluation of a Voluntary Breath-hold Techniquein Patients Receiving Breast Radiotherapy. Clin Oncol (R Coll Radiol) 2017;29(3):e51-e56.

20. Smith BD, Bellon JR, Blitzblau R, Freedman G, Haffty B, Hahn C, Halberg F, Hoffman K, Horst K, Moran J, Patton C, Perlmutter J, Warren L, Whelan T, Wright JL, Jagsi R. Radiation therapy for the whole breast: Executive summary of an American Society for Radiation Oncology (ASTRO) evidence-based guideline. Pract Radiat Oncol 2018;(3):145-52. 